\title{
DEPOTS DE ZIRCONE-ALUMINE OBTENUS PAR PROJECTION PLASMA : FORMATION, MICROSTRUCTURE ET PROPRIETES
}

\author{
M.C. SAINTE-CATHERINE \\ Département de Physique des Surfaces, Centre de Recherches et d'Etudes \\ d'Arcue11, Etablissement Technique Central de l'Armement, 16bis av. \\ Prieur de la cóte d'or, 94114 Arcueil, France
}

\begin{abstract}
Résumé - Des dépôts de zircone-alumine ont êté réalisés par projection plasma d'un mélange à sec de poudre de zircone et de poudre d'alumine. La caractérisation microstructurale et l'utilisation de modèles thermiques ont permis d'interpréter les valeurs de diffusivité thermique obtenues pour ces dépôts.
\end{abstract}

\begin{abstract}
Zirconia-alumina plasma sprayed coatings were made with dry mixing of zirconia powder and alumina powder. The interpretation of the measured thermal diffusivity of zirconia-alumina coatings has been carried out by means of microstructure characterization and thermal models.
\end{abstract}

\section{1 - INTRODUCTION}

Les céramiques à base de zircone ont une importance scientifique et technologique tant d'un point de vue mécanique que thermique. Le système zircone-yttrine trouve des applications structurales importantes en qualité de matériaux frittés et de dépôts obtenus par projection plasma dans le domaine de l'automobile et de l'aéronautique (moteurs diesel et turboréacteurs). Ce système offre des propriétés différentes selon la composition. La zircone tétragonale polycristalline présente une ténacité et une contrainte à la rupture élevées (4-5\% masse $\mathrm{Y}_{2} \mathrm{O}_{3}$ ) en raison du renforcement par transformation de phase (transformation martensitique de la zircone) /1/. La zircone stabilisée de façon plus importante trouve des applications en tant que barrière thermique et plus particulièrement sous la forme de dépôts plasmas pour lesquels une phase tétragonale non transformable procure une certaine stabilité cristallographique au matériau sollicité en température. L'analyse du renforcement des céramiques par dispersion de la phase tétragonale de la zircone $/ 2$ / a permis d'envisager le renforcement de la zircone par un matériau à plus haut module d'Young. Ainsi, à température ambiante, un renforcement significatif de zircones tétragonales polycristallines est obtenu par dispersion de $20 \%$ en masse d'alumine stable /3/. Les concepts de renforcement par dispersion d'une seconde phase ont étê ap̀pliqués aux dépôts plasmas /4/ et aux dépôts par flamme pour lesquels une amélioration de la dureté et de la résistance à l'écaillage est obtenue $/ 5 /$. L'addition d'alumine a été effectuée dans des couches de zircone yttriée élaborées par projection plasma. Cet article présente la corrélation entre les valeurs de diffusivité thermique des couches de zircone-alumine obtenues et leur microstructure.

\section{2 - METHODES EXPERIMENTALES}

Trois poudres céramiques ont été utilisées pour la projection plasma. Il s'agit de l'alumine (granulométrie: $\mathrm{D}_{50}=43 \mu \mathrm{m}, \mathrm{D}_{10}=24 \mu \mathrm{m}, \mathrm{D}_{90}=71 \mu \mathrm{m}$ ), de la zircone yttriée (Zircyt: $\mathrm{ZrO}_{2}-7 \mathrm{Y}_{2} \mathrm{O}_{3}$ masse, granulométrie: $\mathrm{D}_{50}$ $=43 \mu \mathrm{m}, \mathrm{D}_{10}=26 \mu \mathrm{m}, \mathrm{D}_{90}=76 \mu \mathrm{m}$ ), et du mélange à sec des deux poudres précédentes (zircone yttriéealumine: $20 \%$ en masse d'alumine) dans un mélangeur de type Turbula. Les poudres obtenues par atomisation séchage se présentent sous la forme de sphères rugueuses constituées de particules submicrométriques $/ 6 \%$.

Les paramètres de projection sont les suivants:

tension et courant: $50 \mathrm{~V}, 500 \mathrm{~A}$;

gaz plasmagène: mélange $\mathrm{Ar} 15 \%$ vol $\mathrm{H}_{2}$;

distance de projection: $70 \mathrm{~mm}$;

gaz porteur de la poudre: Ar;

épaisseur du dépôt: 300 à $400 \mu \mathrm{m}$.

Le refroidissement des dépôts et la déviation des gaz chauds sont assurés par deux jets d'air comprimé asservis à la torche.

Les poudres céramiques ont été projetées sous air sur un substrat (Inconel 600) revêtu d'une sous-couche en NiCrAlY (déposée sous air; épaisseur: environ $150 \mu \mathrm{m}$ ). 
La microstructure des dépôts a été étudiée par microscopie électronique à balayage (MEB) et en transmission (MET). Des analyses complémentaires par spectrométrie d'émission X (EDS) et par diffraction des rayons $\mathrm{X}$ (rayonnement $\mathrm{CuK} \alpha$ ) ont permis d'identifier les phases présentes. La porosité des couches céramiques a été mesurée par analyse d'images et par une méthode géométrique.

La diffusivité thermique des couches céramiques a été déterminée par la méthode de flash laser $/ 7 /$. Une impulsion (70 J maximum) d'une durée maximale de $450 \mu \mathrm{s}$ est émise à la longueur d'onde $1,06 \mu \mathrm{m}$. L'impulsion est envoyée sur la face avant d'un dépôt décollé de $8 \mathrm{~mm}$ de diamètre et $400 \mu \mathrm{m}$ d'épaisseur. La céramique est recouverte d'un mince film de carbone pour éviter la transmission du rayonnement primaire par l'échantillon. L'image représentative de la distribution de température sur la face arrière de l'échantillon est enregistrée au moyen d'un détecteur InSb $(2-5 \mu \mathrm{m})$ et envoyé sur un oscilloscope digital à mémoíre. La diffusivité du matériau à la température ambiante est calculée de la manière suivante:

$$
a=1,38 \mathrm{~L}^{2} / \mathrm{t}_{1 / 2} \pi^{2}
$$

où $\mathrm{L}$ est l'épaisseur de l'échantillon et $t_{1 / 2}$ le temps requis pour que la face arrière de l'échantillon atteigne la moitié de la valeur maximale de température.

\section{3 - RESULTATS}

3.1 - Microstructure

La figure 1 présente la microstructure d'un dépôt plasma de zircone-alumine brut de projection.

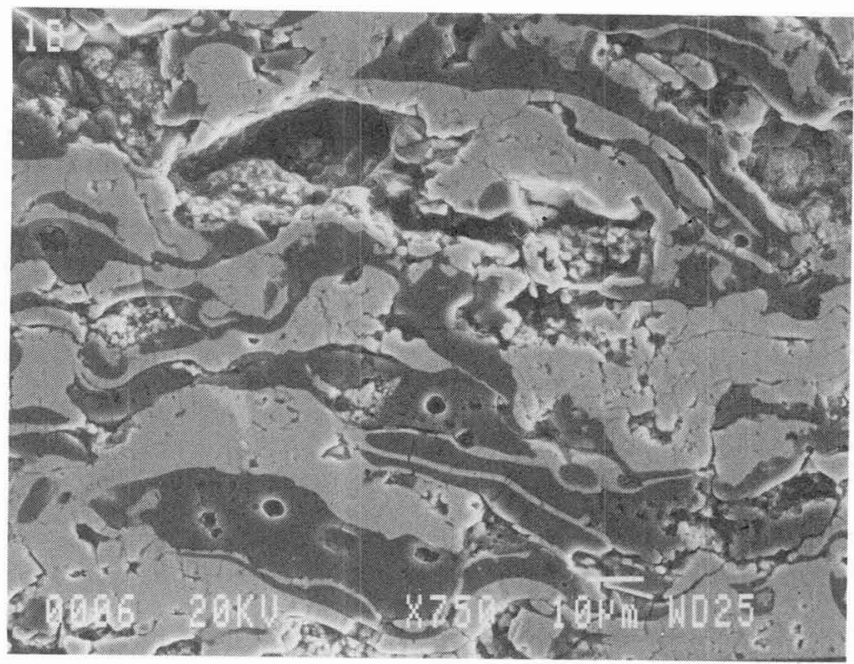

Figure 1: Microstructure d’un dépôt plasma de zircone-alumine (brut de projection, MEB).

Figure 1: Microstructure of zirconia-alumina plasma sprayed coating (as sprayed, SEM).

Le dépôt possède une structure anisotropique lamellaire due à l'utilisation d'un mélange de poudres à sec. Les lamelles de zircone (en clair) et les lamelles d'alumine (en sombre) de quelques micromètres à quelques dizaines de micromètres sont visibles sur la figure 1. Ces lamelles résultent de l'empilement de particules écrasées de même nature. La structure est donc lamellaire à deux échelles. L'aspect lamellaire sous-jacent est caractéristique du mode d'élaboration. Les particules de poudre fondues et accélérées dans le jet de plasma s'écrasent sur la cible où elles se refroidissent à grande vitesse $\left(10^{4} \mathrm{~K}_{\mathrm{s}} \mathrm{s}^{-1}\right)$. A chaque particule fondue correspond une lamelle de quelques micromètres d'épaisseur (figure 1). Cette structure lamellaire est visible sur fractographie de la couche (bas de la figure 1) dans le cas de la microscopie électronique à balayage ou par microscopie électronique en transmission. Sur la figure 2 apparaissent des lamelles de zircone (en sombre) et des lamelles d'alumine (en clair) composées d'une superposition de particules écrasées. Dés espaces interlamellaires sont visibles sur les figures 1 et 2 . Ils sont dus au processus d'écrasement (moindre adhérence de la particule en périphérie). Ils proviennent également de l'emprisonnement d'une mince pellicule de gaz par la particule lors de l'impact. 


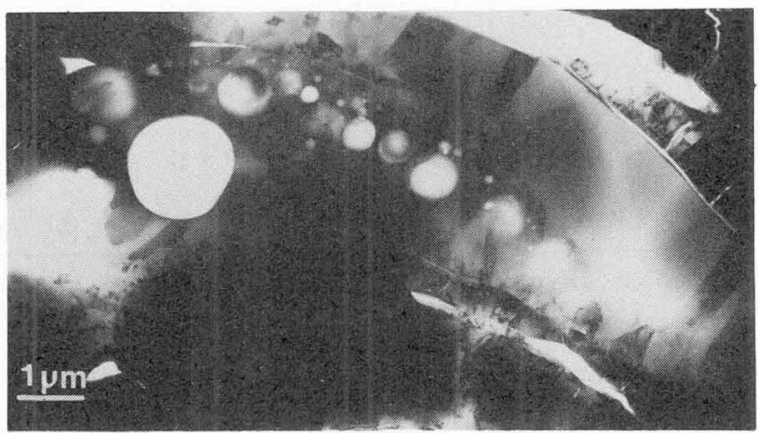

Figure 2: Lamelles de zircone et d'alumine (MET).

Figure 2: Lamellae of zirconia and alumina (TEM).

Outre une structure lamellaire, le dépôt présente une porosité assez importante due essentiellement à la présence d'infondus venant perturber la cohésion de la couche et donnant naissance à des pores de forte taille (quelques micromètres à quelques dizaines de micromètres, figure 1). La figure 3 révèle la microfissuration existant au sein de chaque lamelle et provenant essentiellement de la contraction volumique de la particule au refroidissement.

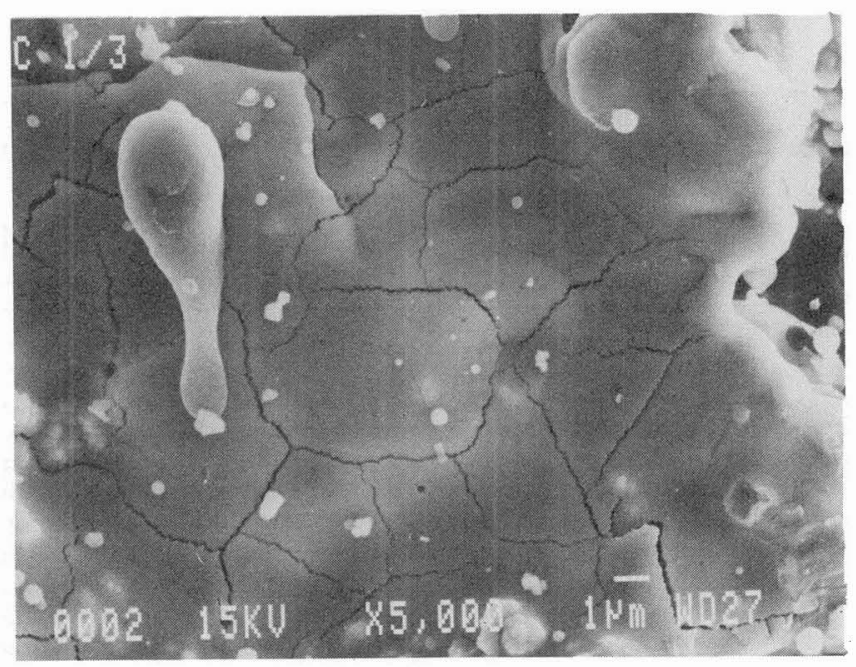

Figure 3: Rêseau de mierofissuration au sein d'une lamelle de zircone (MEB).

Figure 3: Microcrack network in zirconia lamella '(SEM).

3.2 - Cristallographie

Zircone et alumine n'étant pas miscibles à l'état solide $/ 8 /$, les phases cristallographiques présentes dans le dépôt sont propres aux deux constituants. La zircone existe principalement sous la forme tétragonale non transformable $/ 9 /$. Une faible teneur de phase résiduelle monoclinique est décelée. L'alumine se présente essentiellement sous deux formes cristallographiques: l'alumine métastable cubique gamma majoritaire (cette phase est dite trempée au même titre que la phase tétragonale de la zircone) et sous la forme stable rhomboédrique alpha caractéristique de particules non complètement fondues $/ 10 \%$.

3.3 - Diffusivité thermique

Le tableau 1 récapitule les compositions des dépôts, leur porositê et les valeurs de diffusivité thermique moyenne à température ambiante. 
Tableau 1: Composition, porosité et diffusivité thermique des revêtements de zircone-alumine. Table 1: Composition, porosity and thermal diffusivity values of zirconia-alumina plasma sprayed coatings.

\begin{tabular}{cccc}
\hline Echantillon & VZ & VZ20A & A \\
\hline Composilion $\left(\% \mathrm{Al}_{2} \mathrm{O}_{3}\right.$ masse) & 0 & 20 & 100 \\
Porosité, $\mathrm{p}(\% \mathrm{vol})$ & $25 \pm 8$ & $17 \pm 5$ & $36 \pm 10$ \\
Diffusivite $\left(10^{-7} \mathrm{~m}^{2} / \mathrm{s}\right) 298 \mathrm{~K}$ & $3,0 \pm 0,2$ & $3,9 \pm 0,2$ & $3,7 \pm 0,6$ \\
\hline
\end{tabular}

* YZ pour distinguer les dépôts de zircone-yttriée, YZ20A les dépôts de zircone-alumine et A les dépôts d'alumine.

Le tableau 2 indique les caractéristiques thermiques et physiques nécessaires à la compréhension des résultats antérieurs.

Tableau 2: Conductivitê thermique, diffusivitê, chaleur spécifưque et densitê de zircone et alumine denses à température ambiante, de l'air à pression et température ambiante.

Table 2: Thermal conductivity, diffusivity, specific heat and density values of dense zirconia and alumina, of air at ambient pressure and temperature.

\begin{tabular}{|c|c|c|c|c|}
\hline Phase & Zircone & & $\begin{array}{l}\text { Alumine } \\
\text { pure } \alpha\end{array}$ & Alr \\
\hline $\begin{array}{l}\text { Conductivité } \\
(W / m / K)\end{array}$ & $1.8-2.2 / 11 /$ & $\begin{array}{c}\text { pure } \\
p=2,4 \% \text { vol. }\end{array}$ & $35 / 11 /$ & $0,02614 / 11 /$ \\
\hline $\begin{array}{l}\text { Diffusivite } \\
\left(10^{-7} \mathrm{~m}^{2} / \mathrm{s}\right)\end{array}$ & $\begin{array}{l}7 / 11 / \\
8 / 12 / \\
10 / 13 /\end{array}$ & $\begin{array}{c}\mathrm{ZrO}_{2}-\mathrm{MgO} \\
\mathrm{ZrO}_{2}-7 \mathrm{Y}_{2} \mathrm{O}_{3} \\
\text { monocristal } \\
\mathrm{ZrO}_{2}-7 \mathrm{Y}_{2} \mathrm{O}_{3} \\
\mathrm{D}=6 \% \text { \% vol. }\end{array}$ & $80 / 11 /$ & $228 / 11 /$ \\
\hline $\begin{array}{l}\text { Chaleur spéclfique } \\
(\mathrm{J} / \mathrm{kg} / \mathrm{K})\end{array}$ & $\begin{array}{l}455 / 11 / \\
460 / 13 /\end{array}$ & pure & $780 / 11 /$ & $1005 / 11 /$ \\
\hline $\begin{array}{l}\text { Masse spéciflque } \\
\left(10^{3} \mathrm{~kg} / \mathrm{m}^{3}\right)\end{array}$ & $5,75-6,06 / 11 /$ & & $3.99 / 11 /$ & $1,29310^{-3} / 11 /$ \\
\hline
\end{tabular}

\section{4 - DISCUSSION}

Les résultats du tableau 1 indiquent des valeurs de diffusivité sensiblement équivalentes pour un dépôt d'alumine et pour un dépôt de zircone yttriêe contenant $20 \%$ d'alumine (masse). Ces valeurs sont supérieures d'environ $25 \%$ à celle du dépôt de zircone yttriée. Or les valeurs du tableau 2 laissent présager une diffusivité thermique plus importante pour les dépôts à base d'alumine. La composition du matériau ne suffit pas pour expliquer les différences de comportement des couches. Il est donc nécessaire de prendre en compte la porosité des dépôts. La couche d'alumine est environ deux fois plus poreuse que le dépôt YZ20A, ce qui explique le fait que la diffusivité thermique soit sensiblement identique pour les deux couches. La forte porosité obtenue pour ces dépôts est vraisemblablement due à la faible puissance de tír utilisée (25 kW).

Il semble intéressant de calculer la diffusivité du matériau en fonction de la microstructure afin de dégager les facteurs structuraux qui gouvernent les caractéristiques thermiques des dépôts.

Le dépôt plasma de zircone-alumine est un matériau biphasé lamellaire poreux et microfissuré. Les calculs se décomposent essentiellement en quatre étapes correspondant aux différents facteurs microstructuraux (secondes phases: alumine, pores, microfissures, espaces interlamellaires). Un modèle de calcul de conductivité thermique est choisi en fonction de chaque facteur. Par ajustement des valeurs de densité, $\rho$ et de chaleur spécifique, $\mathrm{C}_{\mathrm{p}}$, il est possible de déduire la diffusivité thermique pour une température donnée par la relation:

$$
\mathbf{a}=\kappa / \rho \mathbf{C}_{\mathbf{p}}
$$

4.1 - Conductivité d'un matériau biphasé

La microstructure du matériau YZ20A utilisé dans cette étude consiste en une couche de zircone dans laquelle des lamelles d'alumine ont été dispersées. La formule générale permettant de calculer la conductivité thermique d'un tel type de microstructure est /14/: 


$$
\begin{gathered}
1-V_{D}=\left(\frac{\kappa_{M}}{\kappa}\right)^{a} \frac{\kappa_{D}-\kappa}{\kappa_{D}-\kappa_{M}} \\
\left.a=\frac{F(1-2 F)}{1-(1-F) \cos ^{2} \alpha-2 F\left(1-\cos ^{2} \alpha\right)} \quad b=\frac{1-(1}{\kappa_{M}+b \kappa_{D}}\right)^{c} \\
c=a+\frac{a b(1-F)}{1-2 F}-1
\end{gathered}
$$

Les variables $\kappa, \kappa_{\mathrm{M}}, \kappa_{\mathrm{D}}$ sont respectivement les conductivités thermiques du matériau biphasé, de la matrice et de la phase dispersée; $V_{D}, F$ et $\cos ^{2} \alpha$ sont la fraction volumique, le facteur de forme et le facteur d'orientation de la phase dispersée. La forme des particules est approximée par des ellipsoïdes de révolution dont le facteur de forme est fonction du rapport des axes $z / x$ où $z$ est l'axe de révolution et $x$ le rayon ( $F$ varie de 0 à 0,$5 ; F=0$ dans le cas de lamelles, $F=0,5$ dans le cas d'aiguilles). L'angle d'orientation $\alpha$ est l'angle entre l'axe de révolution et la direction du flux de chaleur. L'intérêt de ce modèle réside dans le fait qu'il tient compte à la fois de la forme des particules et de leur orientation par rapport au flux de chaleur. Il donne d'excellents résultats dans le cas de la zircone partiellement stabilisée (matrice cubique dans laquelle sont dispersês des précipités tétragonaux et monocliniques $/ 15 /$ ).

Les lamelles d'alumine provenant de l'écrasement d'une ou plusieurs particules sont caractérisées par un rapport $z / x=0,10 \pm 0,05$ (le facteur de forme correspondant est de $0,07 \pm 0,03$ ) et un axe de révolution parallèle au flux de chaleur $(\alpha=0)$. La conductivité thermique correspondant au dépôt YZ20A (28\% d'alumine en volume) est de $2,8 \mathrm{~W} / \mathrm{m} / \mathrm{K}$. L 'addition d'alumine sous forme de lamelles dans une matrice de zircone yttriée n'a donc que peu d'influence sur le caractère isolant de la matrice. Une forte augmentation de la conductivité du matériau aurait pu être envisagée compte tenu des valeurs du tableau 2.

Le calcul de la conductivité du matériau biphasé (cas des lamelles perpendiculaires au flux de chaleur) pour des facteurs de formes correspondant aux limites supérieures et inférieures du rapport $z / x$ indique une augmentation de la conductivité avec $F(\kappa=2,717 \mathrm{~W} / \mathrm{m} / \mathrm{K}$ pour $\mathrm{F}=0, \kappa=3,915$ pour $\mathrm{F}=0,3$, échantillon YZ20A). La structure en lamelles opposées au flux de chaleur tend vers une structure à sphères dispersées plus favorable à la conduction. Toutefois, l'erreur qui peut être commise sur l'évaluation de la conductivité à partir de la moyenne du rapport $a / b$ des particules reste faible $(\kappa=2,8 \pm 0,1 \mathrm{~W} / \mathrm{m} / \mathrm{K})$.

La conductivité d'un biphasé (dont la phase dispersée possède un facteur de forme moyen de 0,07 ) est plus sensible à l'orientation des lamelles qu'elle ne l'est à la variation de leur forme. Elle est égale à $5,8 \mathrm{~W} / \mathrm{m} / \mathrm{K}$ lorsque l'orientation des lamelles est de $45^{\circ}$ par rapport au flux de chaleur.

4.2- Conductivité thermique du matériau biphasê poreux.

Le modèle utilisé précédemment peut être appliqué dans le cas d'une structure poreuse car la porosíté peut être assimilée à une seconde phase dispersée dans le matériau. La population de pores est définie par un rapport moyen $x / z=1,8 \pm 0,6$ et une orientation de $35^{\circ} \pm 30$. Les valeurs de la conductivité des matériaux poreux sont regroupées dans le tableau 3 .

Tableau 3: Conductivité thermique calculée de matériaux poreux.

Table 3: Thermal conductivity values of porous materials.

\begin{tabular}{cccc} 
Dépót & $\mathrm{YZ}$ & $\mathrm{YZ20A}$ & $\mathrm{A}$ \\
$\begin{array}{c}\text { Conductivité calculée } \\
(\mathrm{W} / \mathrm{m} / \mathrm{K})\end{array}$ & 1,3 & 2,2 & 17.9 \\
\hline
\end{tabular}

Les pores ont pour effet de diminuer la conductivité thermique des matériaux. Toutefois, les valeurs calculées sont supérieures aux valeurs expérimentales. En effet, la microfissuration des particules constituant le dépôt ainsi que son aspect lamellaire n'ont pas encore été introduits dans le calcul.

4.3 - Conductivité thermique d'un biphasé poreux, microfissuré et lamellaire.

L'analyse d'images effectuée à des grandissements de l'ordre de 1000 ne permet de doser que les pores. Les microfissures et les mauvais contacts interlamellaires sont négligés. La différence entre la porosité globale mesurée par une méthode géométrique et la valeur obtenue par analyse d'images donne accès à leur fraction volumique. Il convient de distinguer les microfissures des espaces interlamellaires. Les premiers sont parallèles au flux alors que les seconds sont perpendiculaires au flux. Sachant que la porosité des dépôts plasmas est principalement ouverte (réseau de pores et fissures interconnectés), la structure d'un matériau poreux microfissuré et/ou lamellaire est de type pénétration. La conductivité d'une telle structure pourrait être calculée par une relation dérivée de la relation utilisée précédemment (cas où $F=0,5$ ) $/ 14 \prime$ Mais ce 
modèle ne convient pas pour une structure lamellaire; il donne des résultats assez proches du modèle relatif à la structure de type dispersion. Ainsi pour le dépôt YZ20A, la conductivité calculée en tenant compte des $3 \%$ de microfissures et espaces interlamellaires est de $2,1 \mathrm{~W} / \mathrm{m} / \mathrm{k}$, valeur identique à celle obtenue en assimilant la porosité totale à une phase dispersée. La contribution des microfissures et des espaces interlamellaires (tableau 4) est évaluée en utilisant respectivement une structure en plaques parallèles et perpendiculaires au flux de chaleur.

La conductivité d'une structure en plaques parallèles est donnée par la relation:

- plaques parallèles au flux de chaleur (résistances thermiques en parallèle)

$$
\kappa=V_{1} \kappa_{1}+V_{2} \kappa_{2} \quad \begin{aligned}
& V_{1}: \text { concentration volumique de la phase } 1 \\
& V_{2}: \text { concentration volumique de la phase } 2 \\
& \kappa_{1}: \text { conductivité de la phase } 1 \\
& \kappa_{2}: \text { conductivité de la phase } 2
\end{aligned}
$$

- plaques perpendiculaires au flux de chaleur (résistances thermiques en série)

$1 / \kappa=V_{1} / \kappa_{1}+V_{2} / \kappa_{2} / 17 /$

Tableau 4: Diffusivités expérimentale et calculée des dépôts de zircone-alumine.

Table 4: Experimental and calculated diffusivity values of zirconia-alumina plasma sprayed coatings.

\begin{tabular}{cccc} 
Echantillion & YZ & YZ20A & A \\
\hline Composition $\left(\% \mathrm{Al}_{2} \mathrm{O}_{3}\right.$ masse) & 0 & 20 & 100 \\
Porosité $(\%$ vol. $)$ & $25 \pm 8$ & $17 \pm 5$ & $36 \pm 10$ \\
Conductivité calculée $(\mathrm{W} / \mathrm{m} / \mathrm{K})$ & 0,66 & 0,97 & 1,22 \\
Diffusivilté calculée $\left(10^{-7} \mathrm{~m}^{2} / \mathrm{s}\right) 298 \mathrm{~K}$ & 3,2 & 4,1 & 6,3 \\
Diffusivité mesurée $\left(10^{-7} \mathrm{~m}^{2} / \mathrm{s}\right) 298 \mathrm{~K}$ & $3,0 \pm 0,2$ & $3.9 \pm 0,2$ & $3,7 \pm 0,6$ \\
\hline
\end{tabular}

La diffusivité des dépôts plasmas est calculée à partir de la conductivité et des valeurs de $\mathrm{C}_{\mathrm{p}}$ et $\rho$ déterminées pour la composition et la porosité des couches. Le calcul progressif de la conductivité du dépôt faisant intervenir à chaque étape un élément microstructural donne des résultats comparables aux valeurs expérimentales. Toutefois, les valeurs calculées sont légèrement supérieures aux mesures. En effet, la teneur en microfissures et en espaces interlamellaires a été estimée d'après les observations microstructurales. Vraisemblablement, le taux d'espaces interlamellaires est plus important. Dans le cas du dépôt d'alumine, une forte valeur calculée de diffusivité est à noter. Les calculs ont été effectués à partir de données relatives à la forme stable de l'alumine. Or la phase majoritaire de l'alumine est la phase métastable cubique. Cette phase se trouve dans un état d'ordre incomplet puisque les lacunes cationiques ne sont pas complètement ordonnées /16/. La conductivité à température ambiante dans le cas des céramiques dépendant du libre parcours moyen des phonons, une conductivitê moindre pour l'alumine cubique est attendue en raișon de la présences de défauts dans le réseau cristallin qui réduisent le lịbre parcours moyen. Cela peut expliquer le fait que la valeur de diffusivité calculée soit supérieure à la diffusivité expérimentale.

\section{5 - CONCLUSION}

Les calculs de diffusivité thermique des dépôts plasmas de zircone-alumine ont êté effectuês en tenant compte de la microstructure et de la cristallographie des couches. Ils ont mis en évidence l'influence prépondérante de l'aspect lamellaire sur les caractéristiques thermiques du matériau comparativement à celle de la composition.

\section{REMERCIEMENTS}

L'auteur remercie le Département de Métallurgie du Centre d'Etudes Nucléaires de Grenoble (France) qui a fourni les poudres et élaboré les dépôts.

Les mesures de diffusivité ont été effectuées au Laboratoire de Thermodynamique de la Faculté des Sciences de Limoges (France) selon les conseils du Professeur C. Martin.

\section{REFERENCES}

/1/ CLAUSSEN N., Advances in Ceramics 12 (1984)

12/ LANGE F.F., J. Mat. Sci. 17 (1982) 225-263 
/3/ FUKUHARA M. J., Am. Ceram. Soc. 72, 2 (1989) 236-242

14/ IWAMOTO N., UMESAKI, ENDO S., MORIMURA T., J. Mat. Sci. 12 (1987) 1113-1119

/5/ KUSHNER B. A., United States Patent 4,588,655 (1986)

16/ COURT M., DANROC J., RANC R., LOMBARD D., $1^{\text {st }}$ Plasma Technik Symposium 2 (1988) 213-220

17/ PAWLOWSKI L., FAUCHAIS P., MARTIN C., Rev. Phys. Appl. 20, 2 (1985) 1-11

18/ ALPER A.M., Science of Ceramics 3 (1967) 339

19/ LANTERI V. HEUER A.H., MITCHELL T.E., Advances in Ceramies 12 (1984) 118-130

110/ LIPPENS B.C., DE BOER J.H., Acta Cryst. 17 (1964) 1312-1321

/11/ TOULOUKIAN Y.S., DEWITT D.P., "Thermophysical properties of matter", IFI/Plenum (1970)

112/ HASSELMAN D.P.H., JONHSON L.F., BENTSEN L.D., SYED R., LEED H.L., SWAIN M.V., Am. Ceram. Soc. Bull. 5 (1987) 799-806

/13/ YOUNGBLOOD G.E., RICE R.W., INGEL R.P., J. Am. Ceram. Soc. 71, 4 (1988) 255-260

/14/ SCHULZ B., High Temp.-High Pressures 13 (1981) 649-660

/15/ BUYKX W.J., SWAIN M.V., Advances in Ceramics 12 (1984) 518-527

/16/ DAUGER A., FARGEOT D., Radia. Eff. 74, 1-4 (1983) 279-289

/17/ CHARVAT F.R., KINGERY W.D., J. Am. Ceram. Soc. 40,9 (1957) 306-315 